\title{
Evaluate and comparission between Software Engineering Methodologies of Agent Systems
}

Asmaa Yaseen Hammo

asmahammo@uomosul.edu.iq

College of Comp. Sciences \$ Math.

\author{
Maher Talal Alasaady \\ maher.alasaady@ntu.edu.iq \\ Northen Technical University
}

\section{University of Mosul}

Accepted 2011/11/02

\author{
Received 2011/07/17
}

\begin{abstract}
Agent based systems software engineering is a new model in the field of software engineering. It provides new approaches for developing agent based systems. Numerous methodologies for agent based systems software engineering have been proposed. However, it is difficult to select a methodology for a specific project, and their application is still limited due to their lack of maturity and weakness for some of them. Evaluating and comparing their strengths and weaknesses is an important step towards developing better methodologies in the future, and help developers to select the most suitable methodology for project development. This research presents an evaluation and comparison of five agent based systems methodologies: Tropos, Gaia, Multi-agent Systems Engineering (MaSE), Multi Agent Systems Development (MASD) and Smart Multi Agent System (SmartMAS) methodology that is suggested by authors. The requirements of an agent based E-Commerce system were studied and applied on the development phases for these five methodologies, and toward a formal study, the evaluation and comparison were performed based upon a framework which addresses four major criteria: concepts and properties, notations and modeling, development process, and pragmatics. The results shows that the proposed methodology is give $(85 \%)$ percentage of covering these criteria for the systems that were developed by using this methodology; this concluded that the proposed methodology is better than other methodologies.
\end{abstract}

Keywords: Agent system, SMART multiagent system, Tropos, Gaia, Multi-agent Systems Engineering (MaSE), Multi Agent Systems Development (MASD)

$$
\begin{aligned}
& \text { تقييم ومقارنة أساليب هندسة برمجيات نظم الوكلاء } \\
& \text { ماهر طلل الأسعدي } \\
& \text { هيئة التعليم التقني / الموصل } \\
& \text { تاريخ قبول البحث: 2011/11/02 } \\
& \text { كلية علوم الحاسوب والرياضيات ياسين حمو امعة الموصل } \\
& \text { تاريخ استلام البحث: 2011/07/17 }
\end{aligned}
$$

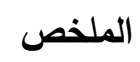


المناسب لمشروع معين هي عملية صعبة، وأيضاً تطبيق هذه الأساليب لا يزال محدوداً بسبب الضعف وعدم

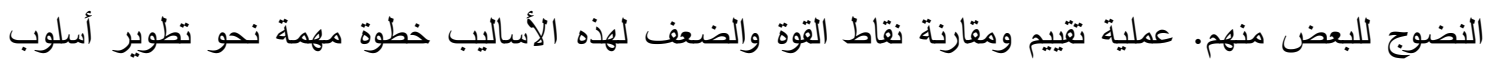

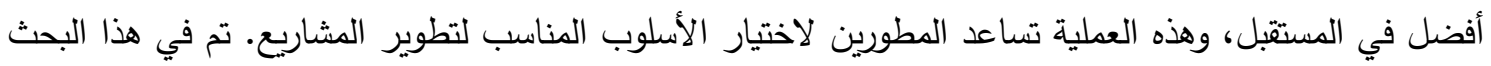
تقييم ومقارنة خمسة أساليب لتطوير الوكلاء وهي Tropos و و Gaia و Multi-agent Systems

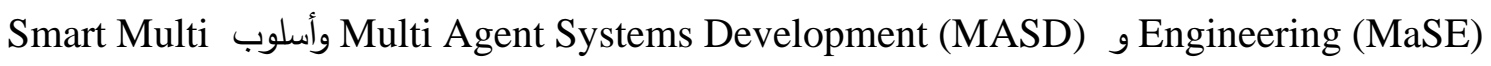
Agent System (SmartMAS)

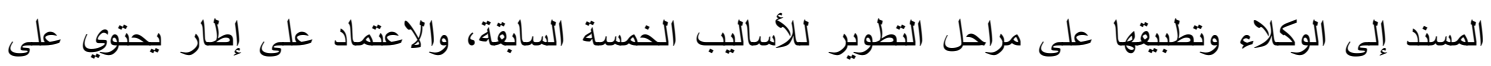
أربعة معايير رئيسية وهي، المفاهيم والخصائص، الترميزات والنمذجة، عملية التطوير ، والناحية التطبيقية. أظهرت نتائج التقييم أن أسلوب SmartMAS يعطي نسبة (85\%) من تغطية هذه المعايير للنظم التي يتم تطويرها

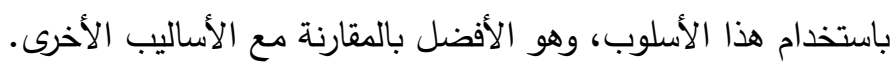

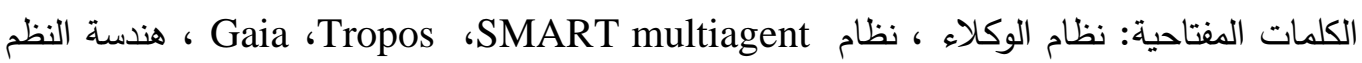
متعددة الوكلاء (MaSE) ، تطوير أنظمة الوكلاء المتعددين (MASD).

1- المقدمة

تتميز تقنية الوكلاء بقدرتها على التعامل مع التعقيد والسلوك الموزع للنظم. لبناء مثل هذه النظم المعقدة، يحتاج المطور إلى أسلوب مناسب كأساس صلب لتطوير النظام بدءاً من مرحلة المتطلبات وصولاً إلى مرحلة البناء. تعرض أساليب هندسة برمجيات نظم الوكلاء معاني جديدة لتحليل وتصميم النظم البرمجية المعقدة، فهذه

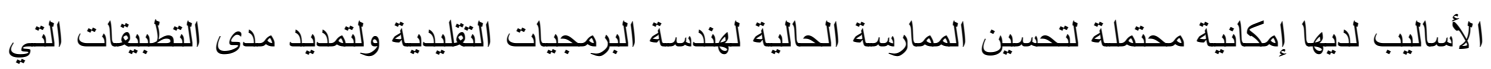
يمكن أن تعالج بشكل أفضل [11]. هناك العديد من الأساليب المقترحة لدعم مهندسي البرمجيات في تطوير نظم

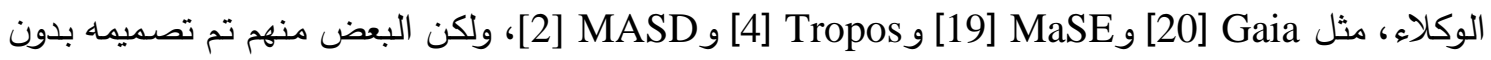

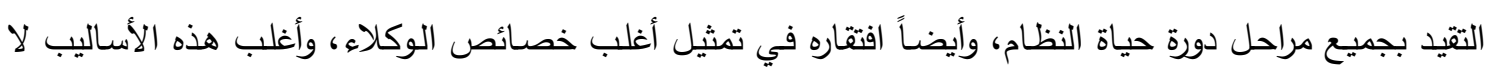

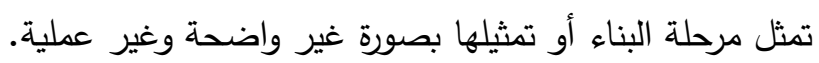

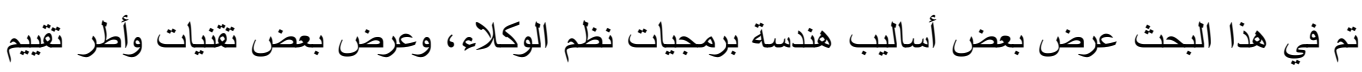

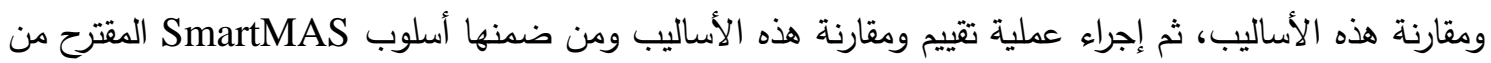
قبل الباحثين [1] لتثخيص نقاط القوة والضعف في هذه الأساليب. ومن خلال دراسة متطلبات نظام التجارة الالكترونية المسند إلى الوكلاء، وتطبيق هذه المتطلبات على مراحل التطوير لكل من الأساليب التي تم عرضها، تم

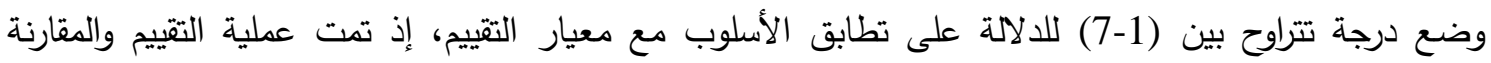

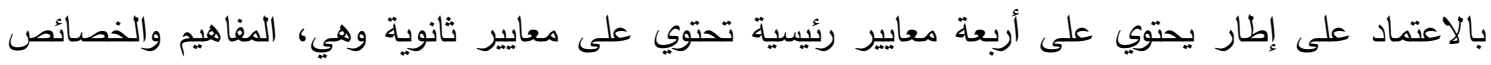
Concepts and Properties

Pragmatics Development Process

2- أهداف البحث الهدف الأساسي للبحث هو تقييم ومقارنة أساليب هندسة برمجيات نظم الوكلاء، إذ تساعد نتائج

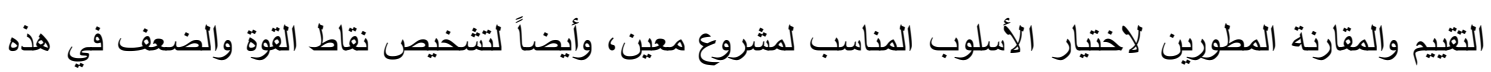
الأساليب نحو تطوير أسلوب أفضل خالٍ من العيوب في المستقبل. 
3- أساليب هندة البرمجيات المعتمدة على الوكلاء ABent Based Software Engineering

Methodologies (ABSE)

ABSE

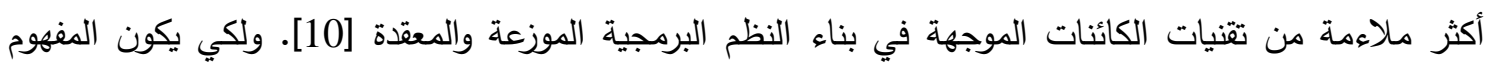
قابلاً للتطبيق، هناك حاجة لإتباع نهجٍ موحدٍ في كل المراحل من خلال دورة حياة تطوير نظام الوكيل والذي يكون

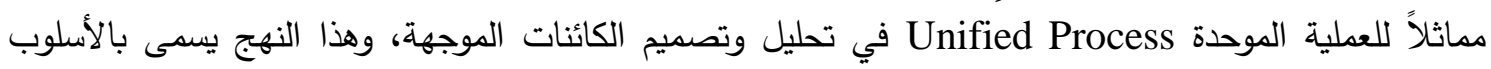
Methodology. يمكن تعريف أسلوب هندسة البرمجيات هو مجموعة منظمة من المبادئ التوجيهية والمفاهيم أو الأنشطة التي تؤدي إلى مساعدة المطورين في القيام في تطوير البرمجيات [9]. دور أساليب تطوير نظم الوكلاء

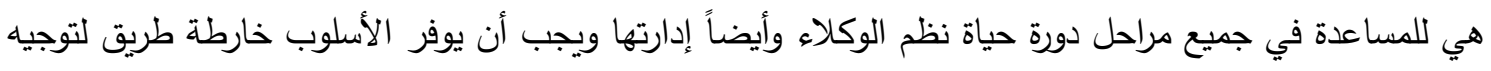
المطورين في إنشاء نظم الوكلاء [18]. هناك عددٌ متزايدٌ من أساليب تطوير الوكلاء والتي تحاول أن تشمل جميع قضايا النظام ومن خلال جميع مراحل تطويره وتتنافس فيما بينها لتكون في المقدمة وتعطي النهج الرئيسي للمطورين.

تم في هذا البحث عرض الأساليب الأكثر شيوعاً لدراستها بثكل نظري من أجل تحديد نقاط القوة

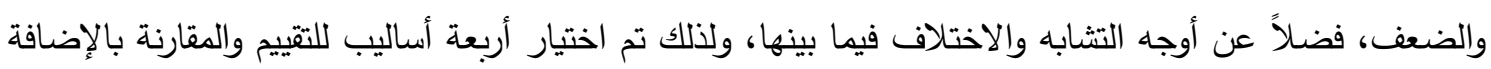
إلى أسلوب مقترح من قبل الباحثين.

Tropos أسلوب 1-3

هو أسلوب لتطوير البرمجيات يتم فيه استخدام أنموذج الوكيل في عملية تطوير النظام. وهو يستخدم رموزاً لتمثيل الوكلاء والأهداف والمهام والتبعيات الاجتماعية لندذجة وتحليل منطلبات النظام بنوعيها المبئية والمتقدمة، والتصميم الهيكلي والتفصيلي [4]. يستند Tropos على فكرتين أساسيتين، الأولى، استخدام مفهوم الوكيل وجميع المفاهيم ذات الصلة (على التى

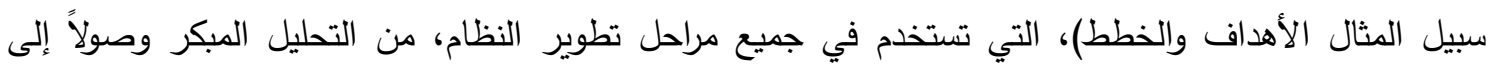
التصميم التفصيلي، الثانية، تغطي المراحل المبكرة جداً من تحليل المتطلبات، وبالتالي تجعل الفهم أعمق للبيئة التي

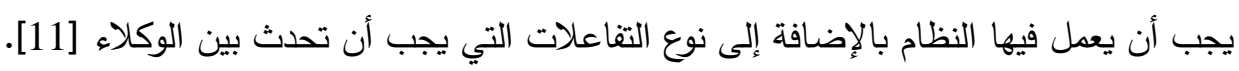

Gaia 2-3 أسلوب

وهو أسلوب لتطوير الوكلاء يعتمد على نمذجة المفاهيم التتظيمية، يقترح أن المطورين يفكرون ببناء نظم الوكلاء على أساس عملية التصميم التظيمي، أي أن التنظيم الحسابي للوكيل يمكن أن يصوّر على شكل مشابه لتتظيم الإنسان والذي يتكون من عدة أدوار مختلفة مع وظائف عديدة تتفاعل مع بعضها البعض التصائ تتقسم المفاهيم

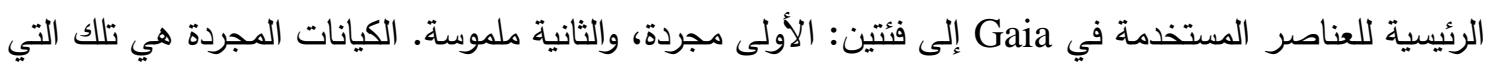

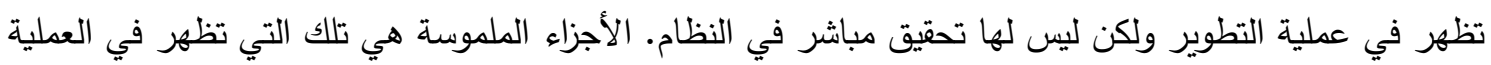
ولديها نسخ مباشرة مثيلة لها في النظام في وقت التشغيل. استتاداً إلى التصنيف المنطقي النطي المذكور أعلاه، تتكون العناصر في النماذج المصممة لمرحلتين رئيسيتين في التطوير : الأولى هي مرحلة التحليل، والتي تستوعب معظم 
المفاهيم المجردة، وتتألف من نمذجة الأدوار وأنموذج التفاعل، الثانية هي مرحلة التصميم، التي تولد العناصر الملموسة وهي تتألف من نموذج الوكيل وأنموذج الخدمة ونموذج التعارف [20]

3aSE أسلوب Ma-3

يوفر هذا الأسلوب المساعدة في تطوير نظم الوكلاء من خلال مرحلتين رئيسيتين وهما: التحليل

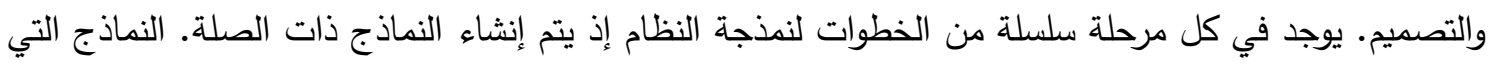
تكونت في الخطوات السابقة يكون ناتج إخراجها مرجعاً لمُدخلات النماذج في الخطوة اللاحقة. التبادلات بين التهاء

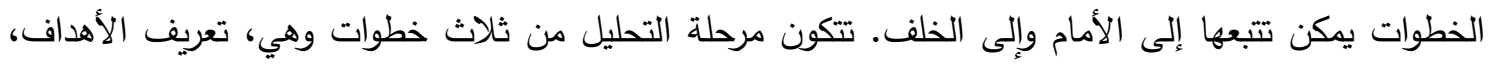

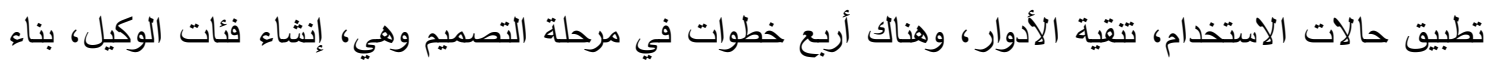

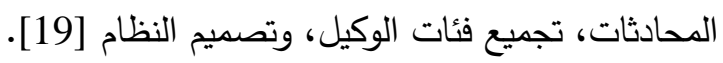

4-3 أسلوب MASD

هو أسلوب لتطوير نظم الوكلاء يتعامل مع مفهوم الوكيل بصورة مجردة وعالية المستوى، يستخدم

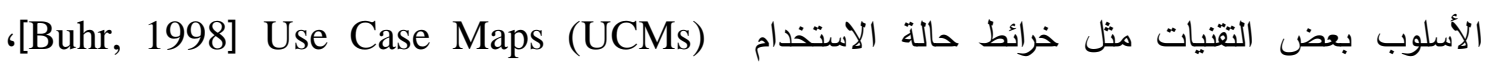
ومخططات حالة استخدام Use Case Diagrams (UCDs)، وأيضاً يدعم هذا الأسلوب البساطة وسهولة الاستخدام وكذلك التتبع. يتألف أسلوب MASD من أربع مراحل رئيسية وهي، مرحلة متطلبات النظام، مرحلة

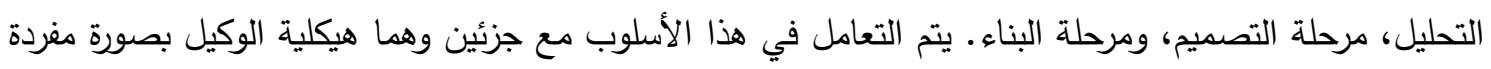
وهيكلية النظام بصورة عامة. تكون نتائج التحليل والتصميم عبارة عن نماذج عديدة للحقائق والأهداف والخطط

\section{5-3 أسلوب SmartMAS}

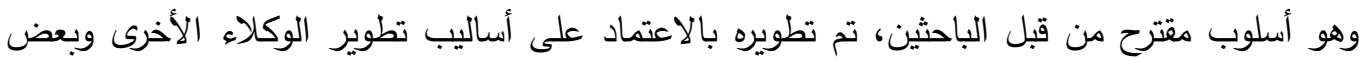

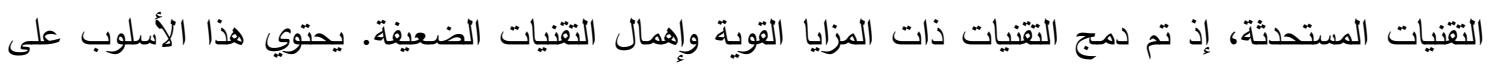
أربع مراحل رئيسية وهي: المتطلبات، التحليل، التصميم، البناء. في مرحلة المتطلبات يوصف النظام بمستوى عالٍ لتكوين مخطط الفاعل، ويستخدم هذا المخطط لوصف النظام والمكونات والمهام التي يجب أن يؤديها كل مكون داخل النظام، ومفهوم النظام من وجهة نظر المستخدم. تقسم مرحلة المتطلبات إلى خطوتين رئيسيتين وهي المتطلبات المبئية والمتقدمة. في الخطوة الأولى يمثل النظام بثكل مخطط بسيط، وفي الخطوة الثانية يتم تفصيل المخطط من خلال: إدخال فاعل النظام، وتجزئة الأهداف، وتكوين المخطط النهائي، وتحليل التبعيات.

الهدف من مرحلة التحليل هو تحويل متطلبات النظام بشكل واضح لتمثيله بشكل مبدئي وتهيئته لمرحلة التصميم، إذ تقوم هذه المرحلة بتحليل متطلبات النظام باستخدام مخطط الفاعل. تقسم مرحلة التحليل إلى خطوتين رئيسيتين: الأولى هي تكوين وصف لهيكلية الوكيل بشكل منفرد، والثانية هي وصف للنفات لنظام بشكل كلي.

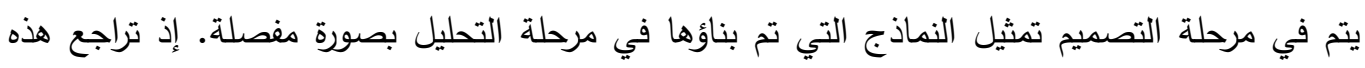

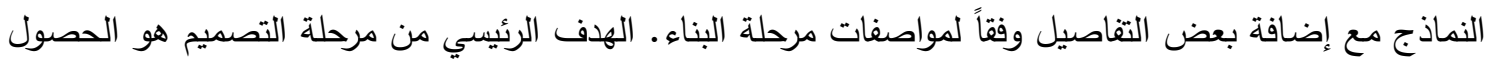

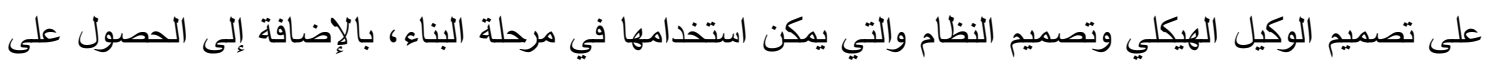


أنماط يمكن إعادة استخدامها في التطبيقات الأخرى المشابهة أو من خلال مراجعتها لغرض التحديث والصيانة والتعديل من قبل المطور، والكثير من فوائد التصميم الأخرى. تتمحور هذه المرحلة في ثلاث خطوات وهي: بناء مخطط التصميم، تعريف حاوية الوكيل، تكوين أنموذج الاتصالات. وأخيراً في مرحلة البناء تجري عملية التطوير الفعلية للنظام من خلال البدء بكتابة الرموز البردجية البهاتة، وبناء النظام على أساس مواصفات مرحلة التصميم. هناك عدة أطر ومنصات لتطوير نظم الوكلاء، والأسلوب المقترح يدعم البعض منها مثل JACK و Jadex، وبعض المنصات الأخرى التي تستخدم هيكلية BDI ومقاييس FIPA مونس

\section{4- تقنيات التقييم Evaluation Techniques}

وهي التقنيات التي تستخدم لغرض تقييم ومقارنة أساليب تطوير البرمجيات. هنالك العديد من التقنيات

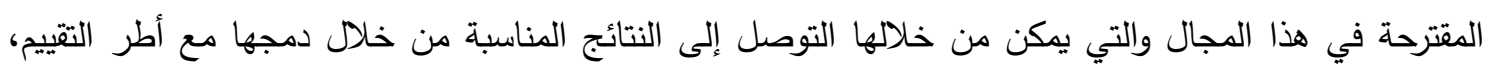

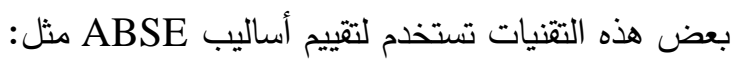

1 المقارنة المستندة على الميزات Feature Based Comparison

وهي المقارنة المستتدة على مجموعة من الميزات التي يتم فحصها في الأساليب، ميزة هذه التقنية هي

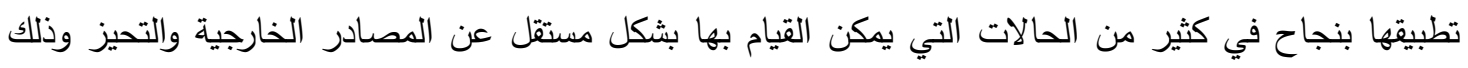

بسبب وضوح هذه الميزات [15].

\section{2. النمذجة الوصفية Meta-Modeling}

يستخدم نهج النمذجة الوصفية لتقييم تحليل وتصميم الأساليب باستخدام نهج رسمي لمقارنة الأساليب. تجري عملية التقييم بالخطوات التالية (1) بناء أنموذج وصفي عام للأسلوب الأساسي (المطلوب) (2) بناء

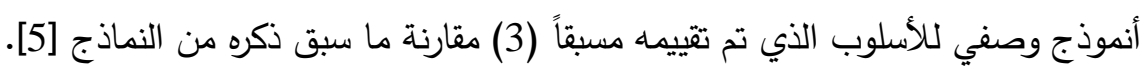

3. Metrics المقاييس

تحلل تقنية المقاييس تعقيد الأسلوب، وهذا يتم من خلال تحليل عدد عناصر البناء، إذ أن عناصر البناء هي البنية التحتية للأسلوب، على سبيل المثال في UML تكون الفئة والكائن والروابط هي أمثلة لعناصر البناء [14]. يقوم هذا الأسلوب باتخاذ القرار على أساس عناصر البناء التي سوف تُحسب وأيضاً درجة تجزئتها،

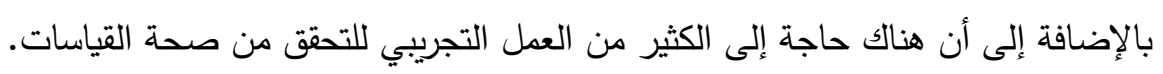

4. تقنيات التقييم التجريبية Empirical Evaluation Techniques

Case وهي تشمل الدراسات الاستقصائية والبحثية والمختبرية والتجارب الميدانية، فضلاً عن دراسات الحالة

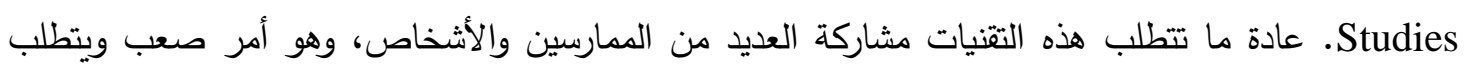
وجود العديد من الموارد. 
5- أطر التقييم Evaluation Frameworks

هنالك العديد من أطر تقييم ومقارنة أساليب ABSE، في كل إطار يتم استخدام تقنية مختلفة، فعلى سبيل المثال، استخدم Sabas إطاراً للتيييم يحتوي على إطار متعدد الأبعاد في كل بعد يوجد معيار للتقييم، على سبيل

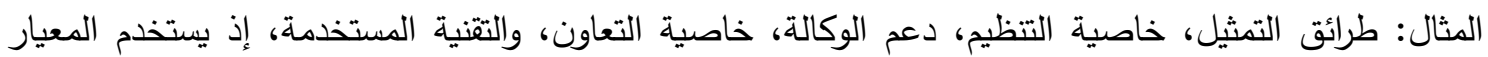

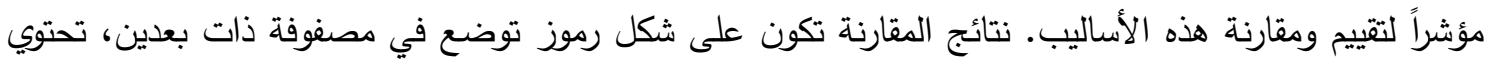

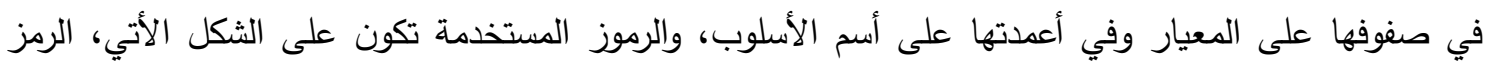

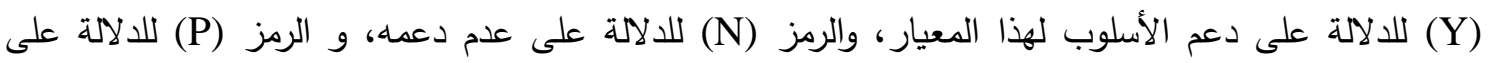

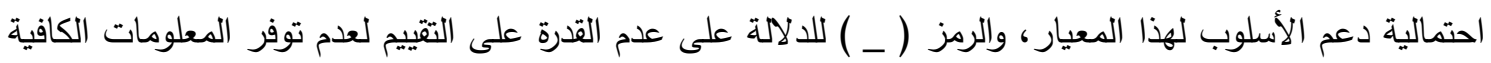

واستخدم Sturm أربعة أبعاد لتجزئة الاختبار إلى أربعة أجزاء. هذه الأبعاد تحتوي على المفاهيم

والخصائص، الترميزات النمذجة، وعملية التطوير ، والناحية التطبيقية [16].

وقام Dam بوضع إطار يحتوي على الأجزاء كما سبق ذكرها، دع تحليل المعيار وقياسه لمختلف الأساليب وإمكانية وضع درجة تتراوح بين (1-7) كقيمة تحدد تطابق هذا المعيار مع الأسلوب المقابل، وفي هذهاء

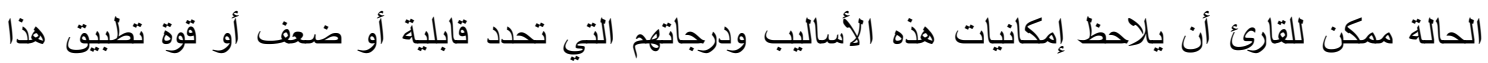
الأسلوب للمعيار ، إذ أن الدرجات الأعلى تدل على دعم الأسلوب للمعيار بصورة أكبر [7]. فيما وضع Shehory إطاراً يستخدم خصائص هندسة البرمجيات التقليدية وخصائص ABSE معياراً لتقييم الأساليب، في كل حقل يوجد معيار مرافق للتقييم، ويستخدم تعليق لفظي لكل معيار مع استخدام الرموز

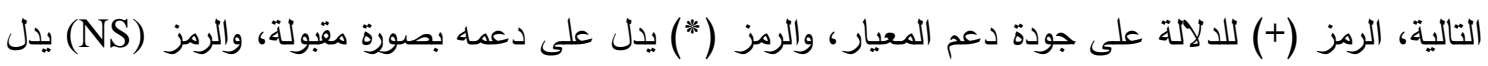
على عدم دعم الأسلوب للمعيار [13].

6- تقييم ومقارنة أساليب هندسة برمجيات نظم الوكلاء

سوف نشير في هذا البحث لأسلوب ABSE على أنه مجموعة كاملة من المبادئ التوجيهية والأنثطة وهي: دورة الحياة الكاملة1، مجموعة شاملة من المفاهيم والنماذج، مجموعة كاملة من التقنيات (القواعد والمبادئ

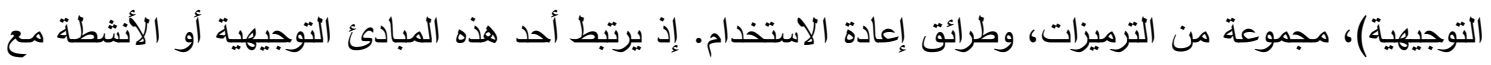
أحد المعايير الرئيسية الأربعة وهي المفاهيم والخصائص، الترميزات والنمذجة، وعملية التطوير ، والناحية التطبيقية. أجريت عملية التقييم والمقارنة بين الأساليب باستخدام التقنية المعتمدة على الميزات بسبب دهيب دعم هذه التقنية

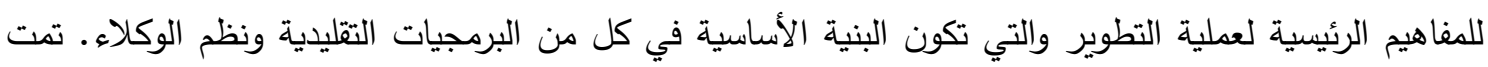
عملية التقييم والمقارنة من خلال ثلاث مراحل، في المرحلة الأولى تم عرض هذه المفاهيم في جدول يوضح

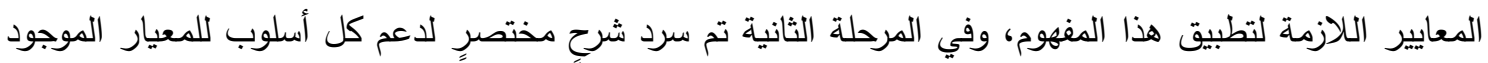

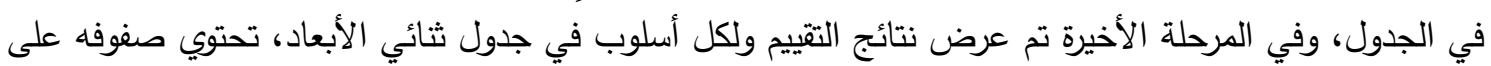
المعايير التي ذكرت في الجدول الأول وتحتوي الأعمدة على أسماء الأساليب المطلوب تقييمها، وفي خلايا الجدول

1 سوف نطلق على دورة الحياة الكاملة للأسلوب الذي يغطي مراحل التطوير الرئيسية وهي المتطلبات والتحليل والتصميم والبناء، دون الأخذ بنظر الاعتبار مراحل الفحص والصيانة والمراحل الأخرى. 
وضعت درجة تتراوح بين (1-7) كقيمة تحدد تطابق هذا المعيار مع الأسلوب المقابل، إذ أن الدرجات الأعلى تدل على دعم الأسلوب للمعيار بصورة أكبر، مع وضع مخطط يصور بشكل واضح النسبة المئوية لـعدل الدرجات الخاصة بكل أسلوب. وضعت الدرجات على أساس دراسة متطلبات نظام التجارة الاكترونية المسند على الوكلاء وتجربته على مراحل التطوير لكل من الأساليب أعلاه، بالإضافة إلى عرض وضلى ومناقئشة النتائج.

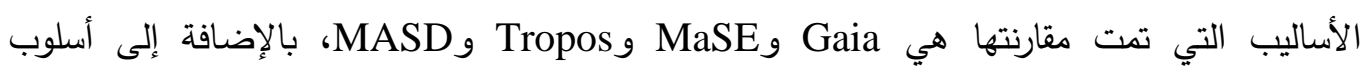

.SmartMAS

Concepts and Properties 1-6 المفاهيم والخصائص

المفهوم هو فكرة مجردة أو فكرة مشتقة أو مستمدة من حالات محددة ضمن نطاق المشكلة، والخاصية هي قدرة خاصة أو ميزة. تتناول هذه الفقرة مسألة ما إذا كان الأسلوب يلتزم بالمفاهيم الأساسية (مفاهيم وخصائص)

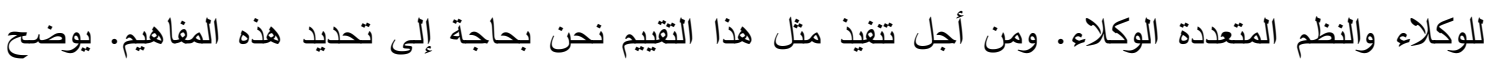
الجدول (1) معايير المفاهيم والخصائص التي يجب أن يتم تقييم أسلوب تطوير الوكلاء على أساسها [17]. الجدول (1). معايير المفاهيم والخصائص

\begin{tabular}{|c|c|}
\hline الوصف & 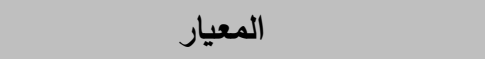 \\
\hline مقدرة الأسلوب لوصف وتعريف الوكلاء بدرجة عالية من التجريد. & تجريد الوكيل Agent Abstraction \\
\hline الرغبات من خلال الوكلاء على الألهواص. العقلانية ولديهم آلية خاصة لتحقيق & $\begin{array}{r}\text { Mental الخواص العقلانية } \\
\text { Properties }\end{array}$ \\
\hline قدرة الوكيل للعمل من دون تدخل خارجي. & الاستقلالية Autonomy \\
\hline مرونة الوكيل للتعامل مع التغييرات الحيوية التي تحصل على البيئة. & التكيف Adaptation \\
\hline تعريف بروتوكول أو آلية معينة للتفاعل بين الوكلاء. & التواصل Communication \\
\hline قدرة الوكيل للتعامل مع الوكلاء الآخرين لتحقيق الأهداف. & التعاون Collaboration \\
\hline مقدرة الوكيل على تنفيذ المهام بصورة متزامنة. & التزامن Concurrency \\
\hline
\end{tabular}

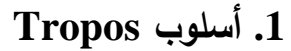

يعرّف الوكلاء من خلال الفاعلين في المخطط التظظيمي. يوجه الوكيل حالاته العقلية ومقدراته من التحليل إلى التصميم التفصيلي باستخدام هيكلية BDI من خلال الأهداف القوية والأهداف الضعيفة والمهام. تكون خواص الاستقلالية والتكيّف مضمّنة في فئة الوكيل. صفة التواصل والتعاون متوفرة في العلاقات التبعية. خاصية

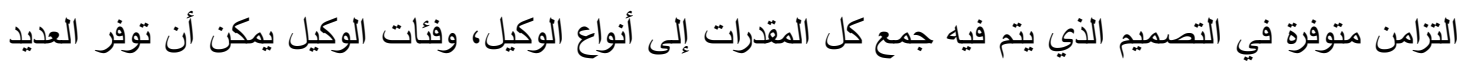
من الخدمات بشكل آني.

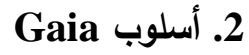

يمثّل الوكيل على شكل دور واحد أو أكثر ويتم نمذجة الدور من خلال استخدام خصائص الحياة

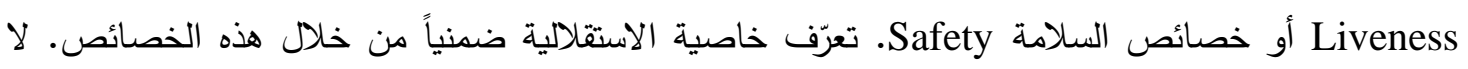
تتوفر الخواص العقلانية في هذا الأسلوب ولكن يتم استخدام خصائص الحياة والسلامة لتوضيح سلوك الوكيل.

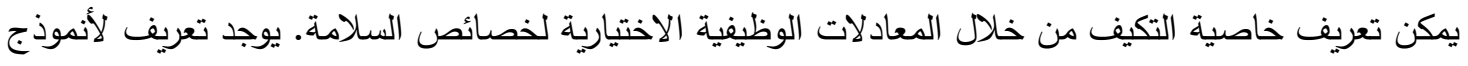


تفاعل الخدمات بين الوكلاء، ولكن لا يوجد تعريف للاتصال بين الوكلاء. خاصية التعاون ممكن أن تتجز من

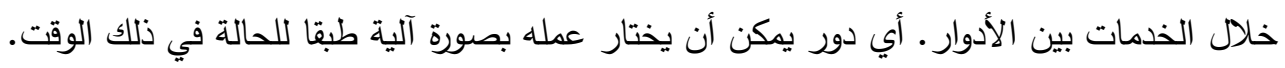

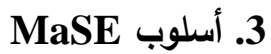

يتم بناء فئات الوكيل من خلال أنموذج الدور . يكون هذا الدور مضمّناً في أهدافه ومهامه المرتبطة. هذه

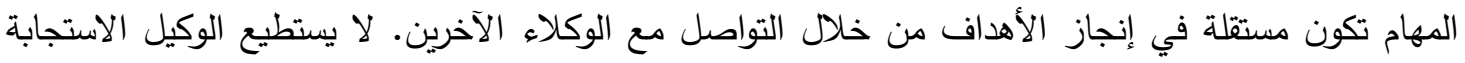

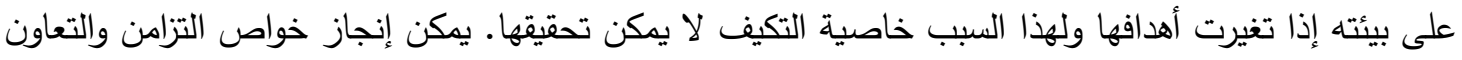
من خلال أنموذج المهام ومن خلال التواصل بين الوكلاء.

4. 1 أسلوب MASD

يتم تعريف الوكيل من خلال الفاعلين في مخططات UCDs والمكونات في مخططات UCMs، وينقل هذا التعريف إلى الدور الذي يكون مضمّناً في أهدافه ومهامه المرتبطة. تعرّف الحقائق والأهداف والخطط في في في نماذج لتثكيل هيكلية BDI. تكون المهام مستقلة في إنجاز الأهداف من خلال الدور . ممكن للوكيل الاستجابة إلى بيئته إذا تغيرت أهدافها وذلك من خلال قيم الحقائق الحيوية.

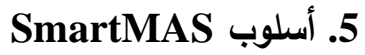

يتم تعريف الوكيل من خلال الفاعلين في مخطط الفاعل ومن خلال تعيين الأدوار المناسبة لهم. تعرّف الحقائق والأهداف والخطط في نماذج لتثكيل هيكلية BDI. يتم تعريف الأهداف، والمهام التي تكون مستقلة في لهافي إنجاز الأهداف من خلال الوكيل. ممكن للوكيل الاستجابة لبيئته إذا تغيرت أهدافها وذلك من خلال قيد قيم الحقائق الحيوية. وجود نموذج للتواصل بين الوكلاء وتعريف التفاعلات. يمكن إنجاز خواص التزامن والتعاون من خلال نموذج الخطط ومن خلال التواصل بين الوكلاء ومن خلال التبعية. يوضح الجدول (2) نتائج تقييم ومقارنة الأساليب لمعايير المفاهيم والخصائص، ويصور الثكل (1) مخطط النسبة المئوية لمعدل نتائج تقييم الأساليب للمفاهيم والخصائص. الجدول (2). نتائج تقييم ومقارنة الأساليب لمعايير الدفاهيم والخصائص

\begin{tabular}{|c|c|c|c|c|c|}
\hline SmartMAS & MASD & Tropos & MaSE & Gaia & \\
\hline 7 & 4 & 7 & 6 & 6 & تجريد الوكيل \\
\hline 7 & 7 & 5 & 2 & 1 & الخواص العقلانية \\
\hline 6 & 6 & 6 & 6 & 6 & الاستقلالية \\
\hline 6 & 6 & 5 & 1 & 4 & التكيف \\
\hline 7 & 7 & 5 & 3 & 4 & التواصل \\
\hline 6 & 6 & 6 & 6 & 6 & التعاون \\
\hline 6 & 6 & 6 & 6 & 6 & التزامن \\
\hline $91 \%$ & $85 \%$ & $81 \%$ & $61 \%$ & $65 \%$ & الأساليب للمفاهيم \\
\hline
\end{tabular}




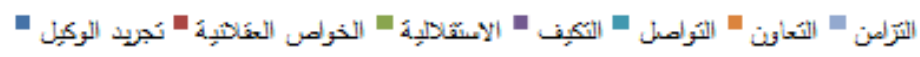

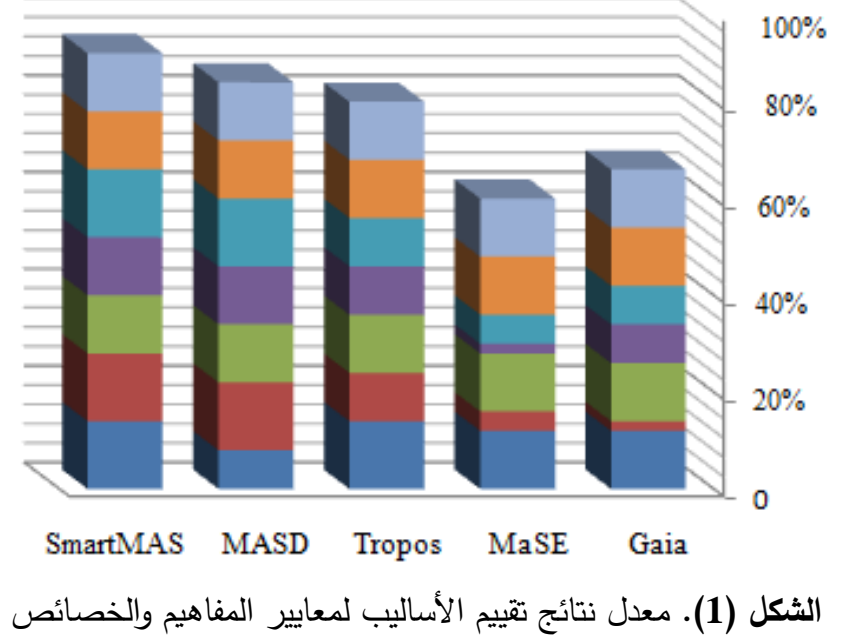

Notations and Modeling 2-5 الترميزات والنمذجة

الترميزات هي الرموز التقنية المستخدمة لتمثيل العناصر داخل النظام. النمذجة هي مجموعة من النماذج

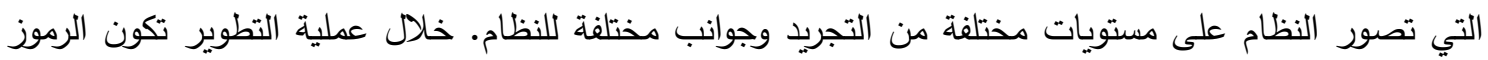

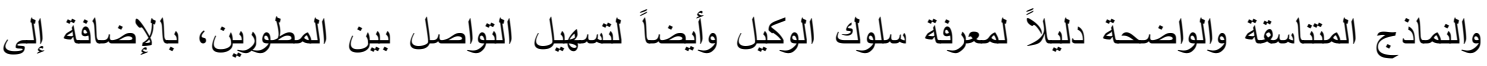
تسهيل التعقيدات من خلال فهمها وبنائها. يوضح الجدول (3) معايير الترميز والنمذجة التي يجب أن يلتزم بها

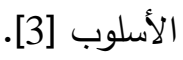

الجدول (3). معايير الترميز والنمذجة

\begin{tabular}{|c|c|}
\hline الوصف & المعيار \\
\hline على استخدام المفاهيم الجديدة. البساطة في فهم واستخدام الأسلوب يعزز قدرات كل من الخبراء والمبتدئين & سهولة الفهم \\
\hline 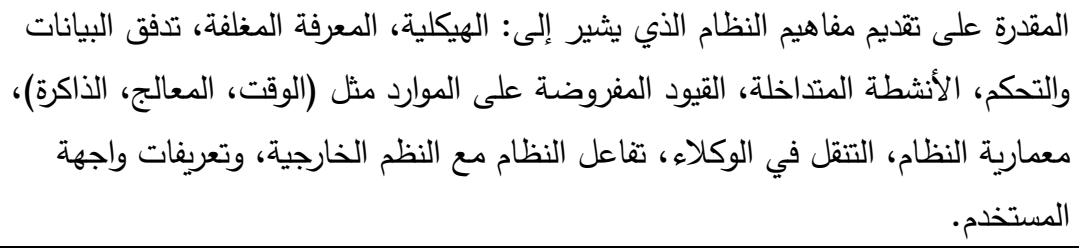 & ق قابلية التعبير \\
\hline 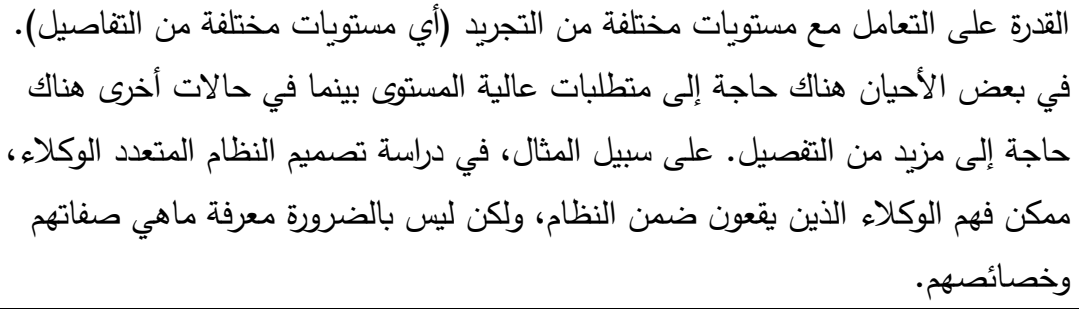 & $\begin{array}{c}\text { إدارة التعقيد } \\
\text { Complexity } \\
\text { Management }\end{array}$ \\
\hline 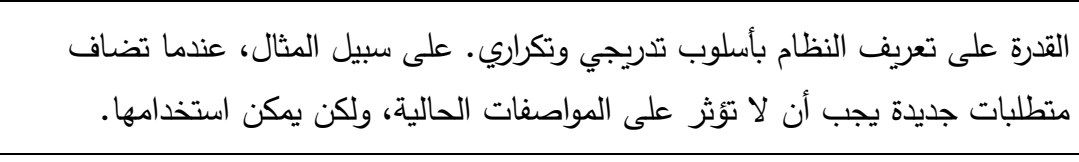 & $\begin{array}{c}\text { التركيبات والتزايدية } \\
\text { Modularity \& } \\
\text { Incremental } \\
\end{array}$ \\
\hline 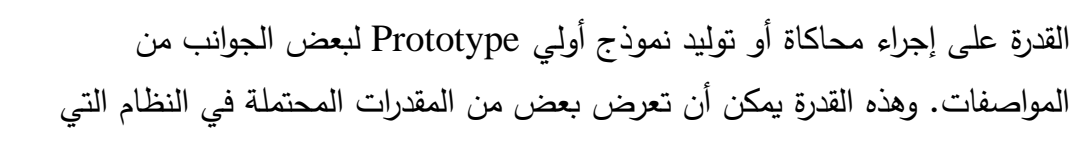 & قابلية التنفيذ والفحص \\
\hline
\end{tabular}




\begin{tabular}{|c|c|}
\hline سوف يتم نمذجتها. & \\
\hline عملية النمذجة التي تقوم بتنقية العوامل إلى كيانات مبسطة لتسهيل التعامل معها. & التقية Refinement \\
\hline المقدرة على تتبع التبعيات بين النماذج. & التبع Traceability \\
\hline هي سمة من سمات عدم الغموض، وهي تتيح للمستخدمين تجنب سوء التفسير للنماذج & Preciseness الدقة \\
\hline
\end{tabular}

Tropos 1

يحتوي هذا الأسلوب على مجموعة من الرسومات التخطيطية الواضحة والسهلة التعبير والتي تجعل من السهل فهم الأسلوب. لا تتوفر الجزئية والتزايدية في مرحلة التحليل، ولكنه يوفر أنماط الهيكلية الاجتماعية في

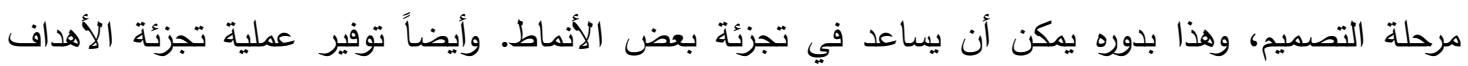

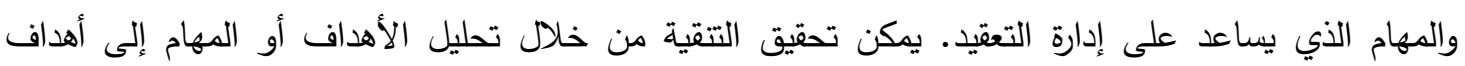
الثانوية أو مهام الثانوية.

Gaia na

يحتوي هذا الأسلوب على نماذج ومخططات جديدة لتدفق السيطرة التي من الممكن أن تعبر عن مفاهيم

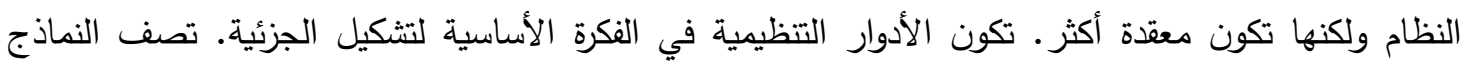

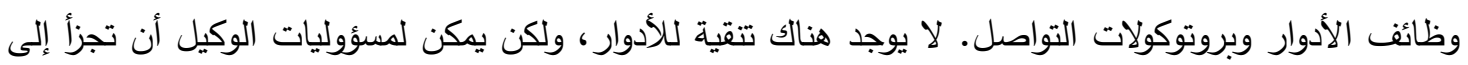

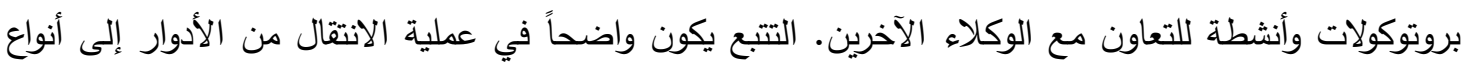
الوكيل.

\section{MaSE 3}

يحتوي هذا الأسلوب على نماذج ومخططات UML في عملية التحليل والتصميم وهذا يدل على قابلية التعبير وسهولة الفهم. يمكن تثكيل الجزئية من خلال تجميع الأهداف والأدوار لبناء أنموذج الأسلوب. عملية التتقية تكون واضحة في نمذجة الأهداف والأدوار • يمكن الحصول على التتبع بسبب الانتقال بين أنموذج الدور وأنموذج فئة الوكيل.

\section{4. أسلوب MASD}

يحتوي هذا الأسلوب على مخططات UCDs و UCMs للتعبير عن المفاهيم في مرحلة المتطلبات،

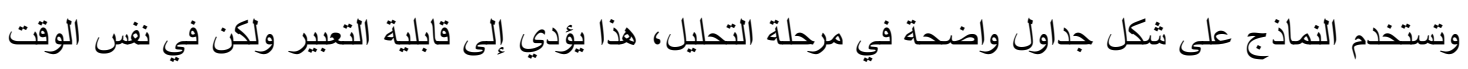
يزيد من التعقيد بسبب كثرة المخططات والنماذج. لا تتوفر في هذا الأسلوب الجزئية والتزايدية في مراحل التحليل والتصميم، ولكنه يوفر أنموذج الأدوار وهذا بدوره يمكن أن يساعد في تجزئة النظام ولكن بدون أنماط. يمكن

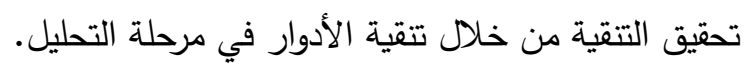




\section{5. أسلوب SmartMAS}

تستخدم الرسومات التوضيحية في مرحلة المتطلبات والأنماط للتصميم الهيكلي الموجودة في أسلوب Tropos، والنماذج في مرحلة التحليل من أسلوب MASD، وهذا بدوره يجمع نقاط القوة في هذه الأساليب وزيادة قوة التعبير وسهولة الفهم. وجود عملية تجزئة الأهداف والمهام يساعد على إدارة التعقيد. تتوفر الجزئية والتزايدية في مرحلة المتطلبات المتقدمة، بالإضافة إلى توفير أنماط الهيكلية الاجتماعية في مرحلة التصميم. يمكن تحقيق التتقية من خلال التحليل المنطقي للأهداف أو المهام إلى الأهداف الثانوية أو المهام الثانوية. يوضح الجدول (4) نتائج تقييم ومقارنة الأساليب لمعايير الترميز والنمذجة، ويصور الثكل (2) مخطط

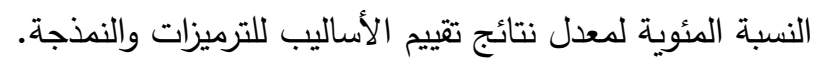

الجدول (4). نتائج تقييم ومقارنة الأساليب لمعايير الترميز والنمذجة

\begin{tabular}{|c|c|c|c|c|c|}
\hline SmartMAS & MASD & Tropos & MaSE & Gaia & \\
\hline 6 & 6 & 6 & 6 & 5 & قابلية التعبير \\
\hline 6 & 4 & 6 & 7 & 2 & سهولة الفهم \\
\hline 6 & 4 & 4 & 6 & 3 & إدارة التعقيد \\
\hline 6 & 3 & 4 & 4 & 6 & التجزئة \\
\hline 3 & 3 & 3 & 1 & 1 & قابلية التنفيذ \\
\hline 5 & 6 & 6 & 6 & 3 & التنقية \\
\hline 6 & 6 & 4 & 5 & 6 & التتبع \\
\hline 5 & 5 & 5 & 5 & 3 & الدقة \\
\hline $76 \%$ & $66 \%$ & $68 \%$ & $66 \%$ & $52 \%$ & الأسلوب تلتيبر \\
\hline
\end{tabular}

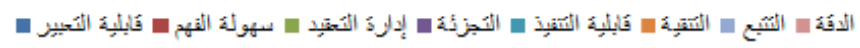

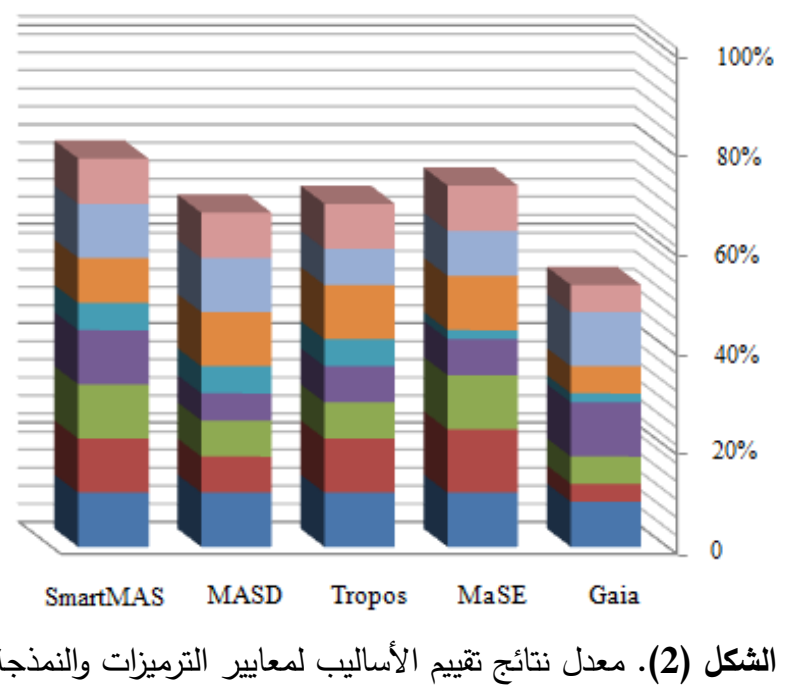


Development Process 3-5 عملية التطوير

عملية التطوير هي عبارة عن سلسلة من الإجراءات والتغييرات والوظائف التي عندما تتجز تكون النتيجة نظاماً محوسباً. وهي تستخدم دليلاً توجيهياً لجميع الفعاليات خلال مراحل التطوير • يوضح الجدول (5) معايير عملية التطوير في الأسلوب [7].

الجدول (5). معايير عملية التطوير

\begin{tabular}{|c|c|}
\hline الوصف & المعيار \\
\hline 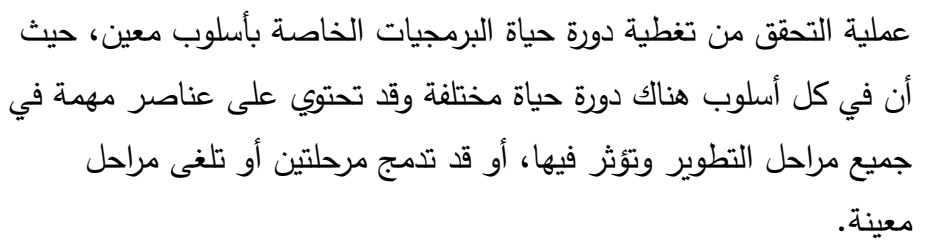 & $\begin{array}{r}\text { تغطية دورة الحياة } \\
\text { Lifecycle coverage }\end{array}$ \\
\hline مقدرة الأسلوب على توفير الطرق لغرض وضع صيغة لتعريف مواصفات & مواصفات النظام \\
\hline الأنماط أو تجميع الأجزاء. & التصميم الهيكلي \\
\hline إعادة الاستخد العدام. Reengineering، وتكوين إذا كان الأسلوب مفيد في تكوين البرامج الجديدة، وعملية إعادة & سياق التطوير \\
\hline توفير الأسلوب على مقترحات بكيفية بناء الوكلاء في النظام. & $\begin{array}{r}\text { أدوات البناء } \\
\text { Implementation Toolkits }\end{array}$ \\
\hline
\end{tabular}

1

خلال دورة حياة النظام لا يغطي الأسلوب مرحلة البناء بصورة تفصيلية. يتعامل هذا الأسلوب مع المتطلبات المبدئية والتي يشارك فيها الزبون لتحديد مواصفات النظام. يحتوي على التصميم الهيكلي الذي يتكون

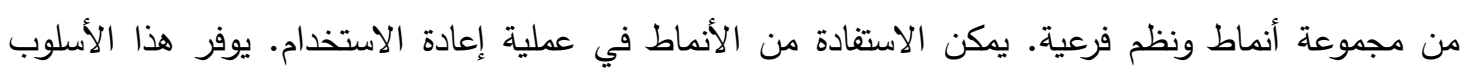

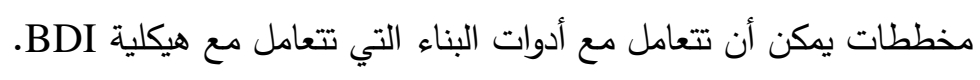

Gaia (أسلوب

يتعامل هذا الأسلوب مع مرحلتي التحليل والتصميم فقط خلال دورة الحياة. يتم التعرف على مواصفات النظام في مرحلة التحليل من خلال تحليل الأدوار • لا يحتوي على مرحلة التصميم الهيكلي. يمكن إعادة استخدام أنواع الوكيل أو الأدوار . لا يعطي تفاصيل بكيفية التعامل مع أدوات البناء.

MaSE

خلال دورة حياة النظام لا يغطي الأسلوب مرحلة المتطلبات ولا يعطي تفاصيل بناء النظام في مرحلة

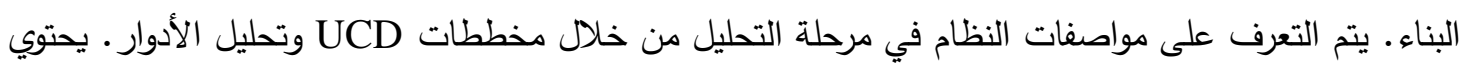
على تفاصيل فئات الوكيل والمحادثات في مرحلة التصميم. يمكن إعادة استخدام فئات الوكيل أو مجاميع فئات 
الوكيل. يعطي الأسلوب صورة واضحة لكيفية التعامل مع بعض أدوات البناء التي تتعامل مع هيكلية الكائنات الموجهة وذلك من خلال استخدامه الفئات.

MASD M. Mلوب

يغطي الأسلوب جميع مراحل دورة حياة النظام الأساسية. يتم التعرف على مواصفات النظام في مرحلة المتطلبات من خلال مخططات UCDs ومخططات UCMs. لا يحتوي هذا الأسلوب على مرحلة التصميم الهيكلي. يمكن الاستفادة من المقدرات في عملية إعادة الاستخدام ولكن بنفس النظام، ومن الصعوبة إعادة الاستخدام لنظم أخرى مشابهة. يوفر MASD نماذج يمكن أن تعطي صورة واضحة لكيفية التعامل مع جميع أدوات البناء التي تتعامل مع هيكلية BDI.

6.

يغطي هذا الأسلوب جميع مراحل دورة حياة النظام الأساسية. يتعامل هذا الأسلوب مع المتطلبات المبدئية

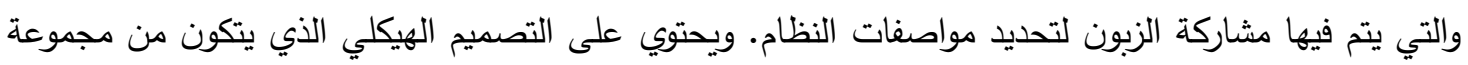

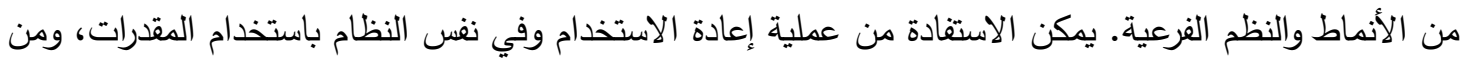

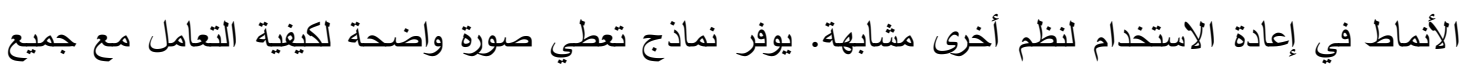
أدوات البناء التي تتعامل مع هيكلية BDI. يوضح الجدولين (6، 7) نتائج تقييم ومقارنة الأساليب لمعايير عملية التطوير ، ونتائج تقييم ومقارنة الأساليب لتغطية دورة حياة النظام على التوالي، ويصور الثكل (3) مخطط النسبة المئوية لمعدل نتائج تقييم الأساليب لتئي لعملية التطوير ·

الجدول (6). نتائج تثييم ومقارنة الأساليب لمعايير عملية التطوير

\begin{tabular}{|c|c|c|c|c|c|}
\hline SmartMAS & MASD & Tropos & MaSE & Gaia & \\
\hline 6 & 6 & 5 & 4 & 4 & تغطية دورة \\
\hline 7 & 5 & 7 & 5 & 5 & مواصفات النظام \\
\hline 6 & 1 & 6 & 5 & 3 & التصميم الهيكلي \\
\hline 5 & 3 & 5 & 6 & 5 & سياق التطوير \\
\hline 7 & 7 & 5 & 6 & 1 & أدوات البناء \\
\hline $\mathbf{8 8 \%}$ & $63 \%$ & $80 \%$ & $74 \%$ & $51 \%$ & التائج تقييم \\
\hline
\end{tabular}

الجدول (7). نتائج تقييم ومقارنة الأساليب لتغطية دورة حياة النظام

\begin{tabular}{|c|c|c|c|c|c|}
\hline SmartMAS & MASD & Tropos & MaSE & Gaia & \\
\hline $\mathrm{Y}$ & $\mathrm{Y}$ & $\mathrm{Y}$ & $\mathrm{N}$ & $\mathrm{N}$ & المتطلبات \\
\hline $\mathrm{Y}$ & $\mathrm{Y}$ & $\mathrm{Y}$ & $\mathrm{Y}$ & $\mathrm{Y}$ & التحليل \\
\hline $\mathrm{Y}$ & $\mathrm{Y}$ & $\mathrm{Y}$ & $\mathrm{Y}$ & $\mathrm{Y}$ & التصميم \\
\hline
\end{tabular}




\begin{tabular}{|c|c|c|c|c|r|}
\hline Y & Y & No Details & No Details & N & البناء \\
\hline N & N & N & N & N & الفحصانة \\
\hline N & N & N & N & N & (الصصانة \\
\hline
\end{tabular}

Pragmatics 4-5 الناحية التطبيقية

تثير الناحية التطبيقية إلى التعامل مع الجوانب العملية لاستخدام الأسلوب. تتتاول هذه الفقرة الناحية التطبيقية لاعتماد الأسلوب لمشروع معين أو داخل المؤسسة، يوضح الجدول (8) المعايير اللازمة من الناحية التطبيقية [7].

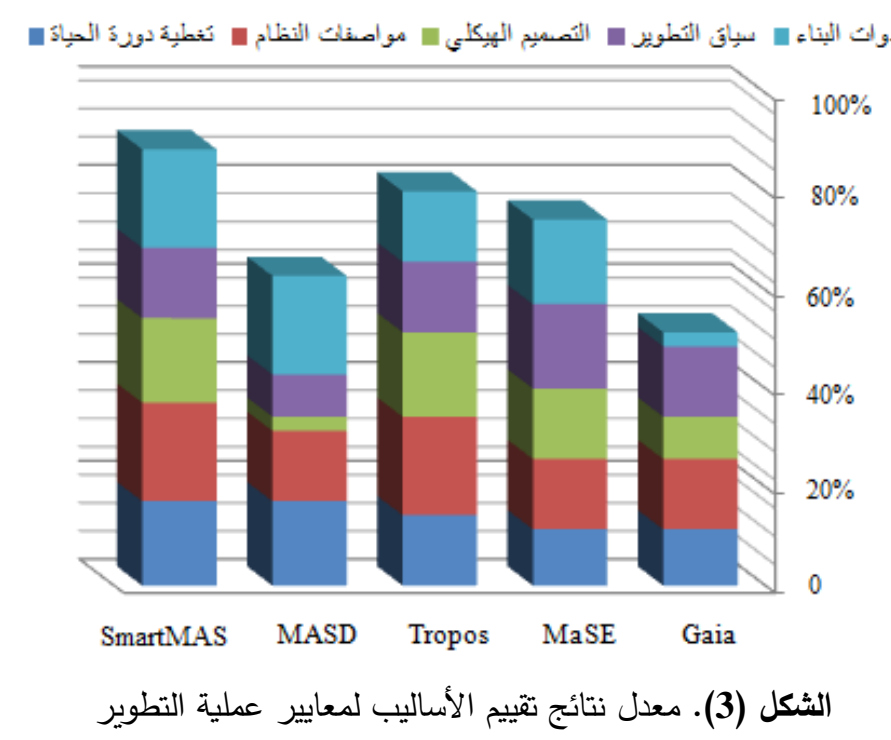

الجدول (8). معايير الناحية التطبيقية

\begin{tabular}{|c|c|}
\hline الوصف & المعيار \\
\hline 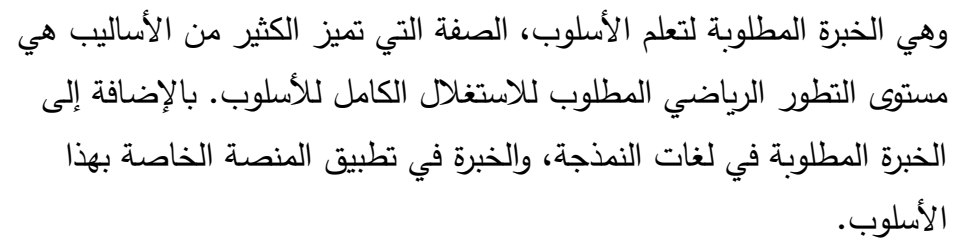 & $\begin{array}{r}\text { لخبرة المطلوبة } \\
\text { Required Expertise }\end{array}$ \\
\hline هل استخدام الأسلوب مناسب لهيكلية معينة؟ على سبيل المثال BDI. & $\begin{array}{r}\text { لنمذجة المناسبة } \\
\text { Modeling Suitability }\end{array}$ \\
\hline هل هل استخدام الأسلوب مناسب لمجال تطبيق معين؟ على سبيل المثال في الوقت & $\begin{array}{r}\text { طاق التطبيق } \\
\text { Domain applicability }\end{array}$ \\
\hline ماهي الأدوات والموارد المتوفرة التي تستخدم مع الأسلوب في جميع المراحل. & $\begin{array}{r}\text { لأدوات المتوفرة } \\
\text { Tools available } \\
\end{array}$ \\
\hline 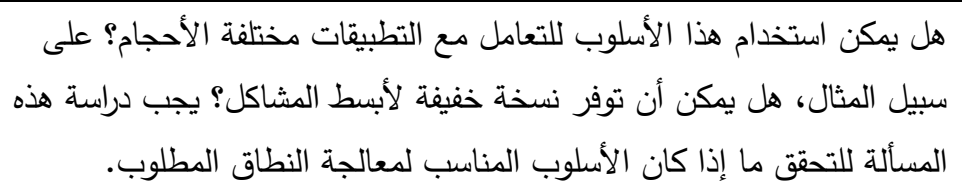 & لتوسيع Scalability \\
\hline
\end{tabular}




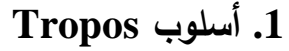

بسبب عدم ذكر التفاصيل الكافية لمرحلة البناء يكون من الصعب تتفيذ النظام بالإضافة إلى الحاجة للخبرة المناسبة لتطوير نظام بصورة جيدة. بالرغم من تعامل هذا الأسلوب مع مخططات UML في مرحلة التصميم، لئل ولكن لا يوجد هناك أدوات متوفرة لتساعد المصممين في مرحلة التحليل وجزء من مرحلة التصميم.

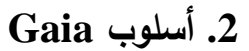

يحتاج هذا الأسلوب إلى الخبرة الكافية لغرض فهم خصائص الحياة والسلامة وتحويلها إلى صيغة تنفيذية. ولكنه لا يحتوي على أدوات للتحليل والتصميم، وهذا يجعل المصممين يبحثون عن طريقة مناسبة لإكمال المخططات بأنفسهم وهذا يتحقق من خلال خصائص الحياة والسلامة.

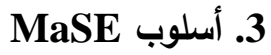

لا يحتاج هذا الأسلوب إلى خبرة كبيرة وذلك لأنه يتعامل مع مخططات UML. يتعامل مع أداة الوكيل Agent Tool بصورة آلية، وعملية تدقيق النماذج [8].

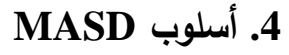

في مرحلة البناء يتم وصف النظام بصورة تفصيلية وهذا يؤدي إلى سهولة التعامل مع أدوات التطوير وعدم

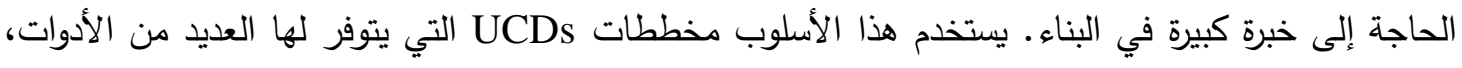
ولكن لا يحتوي على أدوات لمخططات UCMs في مرحلة المتطلبات أو أدوات في مراحل التحليل والتصميم.

\section{1 أسلوب SmartMAS}

في مرحلة البناء يتم وصف النظام بصورة تفصيلية وهذا يؤدي إلى سهولة التعامل مع أدوات التطوير وعدم الحاجة إلى خبرة كبيرة في البناء. لا يحتوي هذا الأسلوب على أدوات للتحليل والتصميم.

يوضح الجدول (9) نتائج تثييم ومقارنة الأساليب لمعايير الناحية التطبيقية، ويصور الثكل (4) مخطط

النسبة المئوية لمعدل نتائج تقييم الأساليب للناحية التطبيقية.

الجدول (9). نتائج تقييم ومقارنة الأساليب لمعايير الناحية التطبيقية

\begin{tabular}{|c|c|c|c|c|c|}
\hline SmartMAS & MASD & Tropos & MaSE & Gaia & \\
\hline 6 & 6 & 4 & 7 & 2 & الخبرة المطلوبة \\
\hline 7 & 7 & 7 & 3 & 3 & النمذجة المناسبة \\
\hline 6 & 6 & 6 & 6 & 6 & نطاق التطبيق \\
\hline 5 & 5 & 5 & 7 & 1 & الأدوات المتوفرة \\
\hline 6 & 4 & 6 & 5 & 5 & التوسيع \\
\hline $86 \%$ & $80 \%$ & $80 \%$ & $80 \%$ & $48 \%$ & الأسلوب للنائج تقييم \\
\hline
\end{tabular}




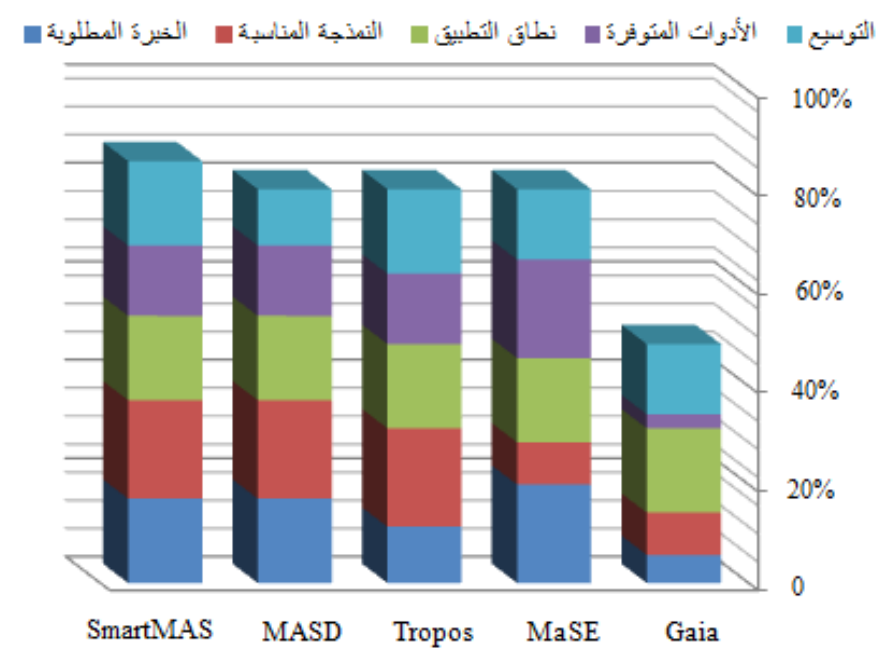

الثكل (4). معدل نتائج تقييم الأساليب لمعايير الناحية التطبيقية

6- الخلاصة والاستنتاجات

تم في هذا البحث عرض بعض أساليب ABSE، ثم عرض تقنيات وأطر تقييم ومقارنة هذه الأساليب، ثم

إجراء عملية تقييم ومقارنة خمسة أساليب لتطوير نظم الوكلاء من ضمنها أسلوب مقترح من قبل الباحثين من خلال

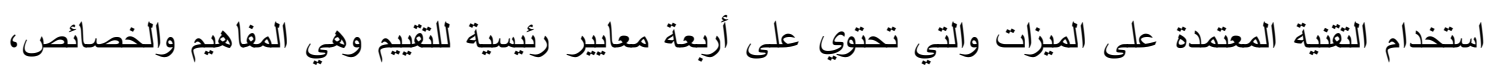
الترميزات والنمذجة، عملية التطوير، والناحية التطبيقية، وتم استخدام إطار يحتوي في صفوفه التهنه على معايير التقييم

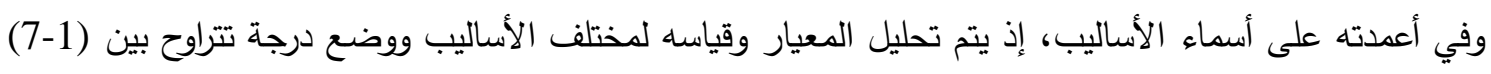
كقيمة تحدد تطابق هذا المعيار مع الأسلوب المقابل، تم وضع الدرجات على أساس دراسة متطلبات نظام التجارة الالكترونية المسند على الوكلاء وتجربته على مراحل التطوير لكل من الأساليب السابقة. يوضح التحلى الجدول (10) نتائج تقييم الأساليب، ويصور الثكل (5) معدل نتائج تتييم الأساليب لجميع المعايير. نلاحظ أن الأسلوب المقترح يعطي نسبة (85\%) من تغطية هذه المعايير للنظم التي يتم تطويرها باستخدام هذا الأسلوب، وهو الأفضل بالمقارنة مع الأساليب الأخرى. هذه النتائج تساعد المطورين لاختيار

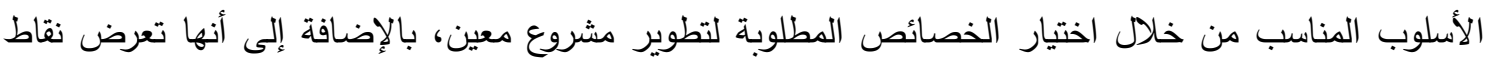
القوة والضعف في هذه الأساليب مما يساعد في تطوير أسلوب خالي من العيوب في المستقبل. الجدول (10). نتائج تقييم الأساليب

\begin{tabular}{|c|c|c|c|c|c|}
\hline SmartMAS & MASD & Tropos & MaSE & Gaia & \\
\hline $91 \%$ & $85 \%$ & $81 \%$ & $61 \%$ & $65 \%$ & والخصائص تقييم الأسلوب للمفاهيم \\
\hline $76 \%$ & $66 \%$ & $68 \%$ & $66 \%$ & $52 \%$ & والنتائج تقييم الأسلوب للترميزات \\
\hline $88 \%$ & $63 \%$ & $80 \%$ & $74 \%$ & $51 \%$ & نتائج تقييم الأسلوب لعملية التطوير \\
\hline $86 \%$ & $80 \%$ & $80 \%$ & $80 \%$ & $48 \%$ & التطبئج تقييم الأسلوب للناحية \\
\hline
\end{tabular}




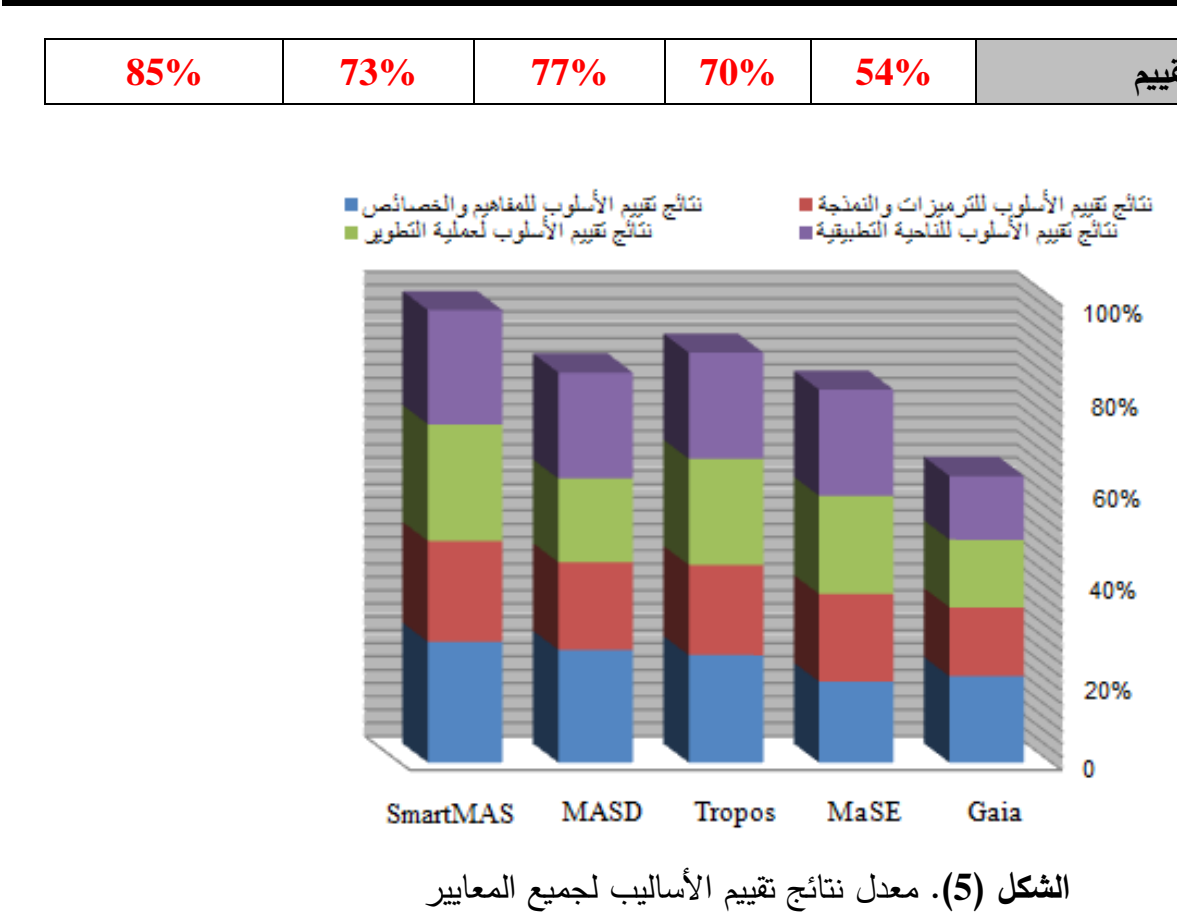

7- - الأعمال المستقبلية

بالنسبة للأعمال المستقبلية هناك ناحيتان لتطوير العمل في هذا البحث:

1. أساليب ABSE في تطور دائم ولذلك فأن من المهم إجراء التقييم والمقارنة للأساليب الجديدة مع بعضئان البعض ومع الأساليب القديمة لتحديد الأسلوب الأفضل والاتجاه نحو أسلوب موحد يحمل جمئ الجراه لفيع نقاط القوة، ولذلك فأن هذا العمل يتم تكراره في المستقبل. 2. عملية تقييم تعقيد الأساليب من خلال حساب تعقيد العناصر البنائية التي يطورها الأسلوب وبالاعتماد على في البيانات الإحصائية التي يتم حسابها من خلال المعادلات. 


$$
\begin{aligned}
& \text { المصادر } \\
& \text { حمو، أسماء ياسين، الأسعدي، مـاهر طلال، 2011، "أسلوب مقترح لهندسة برمجيات نظم الوكلاء"، }
\end{aligned}
$$

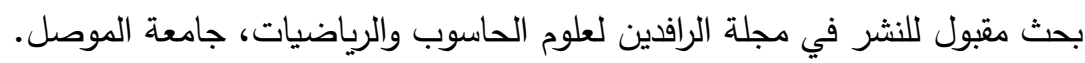

[2] Abdelaziz, T., Elammari, M., and Branki, C., 2008, "MASD: Towards a Comprehensive Multi-agent System Development Methodology" SpringerVerlag Berlin Heidelberg, pp. 108-117.

[3] Ardis, M., Chaves, J., Jagadeesan, L., Mataga, P., Staskauskas, M., Olnhausen, J., 1996, "A Framework for Evaluating Specification Methods for Reactive Systems, Experience Report", IEEE Trans. Software Engineering, Vol. 22, No. 6, pp 378-389.

[4] Bresciani, P., Giorgini, P., Hiunchiglia, F., Mylopoulos, J., Perini, A., 2001, "TROPOS: An Agent-Oriented Software Development Methodology", Technical Report \#DIT-02-0015, AAMAS Journal.

[5] Brinkkemper, S., Hong, S., Goor, G., 1993, "A formal approach to the comparison of object-oriented analysis and design methodologies", in Proc.of the Twenty-Sixth Hawaii Intl. Conf., Vol. 4 , pp. 689-698.

[6] Buhr, R., 1998, "Use Case Maps as Architectural Entities for Complex Systems", IEEE Transactions on Software Engineering vol. 24(12), pp.11311155.

[7] Dam, K., Winikoff, M., 2003, "Comparing Agent-Oriented Methodologies", Fifth International Bi-Conference Workshop on Agent-Oriented Information Systems(AOIS-2003), Melbourne, Australia, at AAMAS'03, pp. 78-93.

[8] DeLoach, S., 2001, "Analysis and Design using MaSE and agent Tool", Proceedings of the 12th Midwest Artificial Intelligence and Cognitive Science Conference (MAICS 2001). Miami University, Oxford, Ohio, pp. 45-57.

[9] Jeffrey M., 1997, "An introduction to software agents", Jeffrey M. Bradshaw, editor, Software Agents, AAAI Press / The MIT Press, vol. 43,3 p.

[10] Lind, J., 2000, "Issues in agent-oriented software engineering", In P. Ciancarini and M. Wooldridge, editors, AOSE, volume 1957 of LNCS, Springer, pp. 45-58.

[11] Parandoosh, F., 2007, "Evaluating Agent-Oriented Software Engineering Methodologies", IEEE International Workshop on Soft Computing Applications, Gyula, Hungary - Oradea, Romania, pp 21-23.

[12] Sabas, A., Badri, M., Delisle S., 2002, "A Multidimentional Framework for the Evaluation of Multiagent System Methodologies", Proceedings of the 6th World Multiconference on Systemics, Cybernetics and Informatics (SCI-2002), Orlando (Florida, USA), Volume I, pp. 211-216.

[13] Shehory, O., Sturm, A., 2001, "Evaluation of Modeling Techniques for AgentBased Systems", AGENTS01, Montreal, Quebec Canada, p.91-103.

[14] Siau, K., Rossi, M., 1998, "Evaluation of Information Modeling Methods - A Review", in Proc. 31 Annual Hawaii International Conference on System Science, pp. 314-322. 
[15] Siau, K., Cao, Q., 2001, "Unified Modeling Language: A Complexity Analysis, Journal of Database Management", Vol. 12, No. 1, pp. 26-34.

[16] Sturm, A., Shehory, O., 2003, "A Framework for Evaluating Agent-Oriented Method-ologies", Fifth International Bi-Conference Workshop on AgentOriented Information System (AOIS-2003), Springer, pp. 94-109.

[17] Tran Q., Low G., Williams M., 2003, "A Feature Analysis Framework for Evaluating Multi-agent System Development Methodologies", ISMIS 2003, pp. 613-617.

[18] Tveit, A., 2001, "A survey of Agent-Oriented Software Engineering", First NTNU CSGSC.

[19] Wood, M., DeLoach, S., 2001, "An Overview of the Multi-agent Systems Engineering Methodology", in Agent-Oriented Software Engineering. P. Ciancarini, M. Wooldridge, (Eds.) Lecture Notes in Computer Science. Vol. 1957, Springer Verlag, Berlin.

[20] Wooldridge, M., Jennings, N. R. and Kinny, D., 2000, "The Gaia Methodology for Agent-Oriented Analysis and Design", Journal of Autonomous Agents and Multi-Agent Systems, vol. 3, pp. 285-312. 